\title{
Cost Effectiveness of Itraconazole in the Prophylaxis of Invasive Fungal Infections
}

\author{
Robin de Vries, ${ }^{1}$ Simon Daenen, ${ }^{2}$ Keith Tolley, ${ }^{3}$ Axel Glasmacher, ${ }^{4}$ Archie Prentice, ${ }^{5}$ \\ Sarah Howells, ${ }^{3}$ Hariette Christopherson, ${ }^{3}$ Lolkje T.W. de Jong-van den Berg ${ }^{1}$ and \\ Maarten J. Postma ${ }^{1}$ \\ 1 Groningen Research Institute of Pharmacy (GRIP), University of Groningen, Groningen, \\ The Netherlands \\ 2 University Medical Centre Groningen (UMCG), Groningen, The Netherlands \\ 3 Ortho Biotech, High Wycombe, UK \\ 4 University Hospital Bonn, Bonn, Germany \\ 5 Derriford Hospital, Plymouth, UK
}

\section{Abstract}

Background: Invasive fungal infections in neutropenic patients treated for haematological malignancies are associated with a high mortality rate and, therefore, require early treatment. As the diagnosis of invasive fungal infections is difficult, effective antifungal prophylaxis is desirable. So far, fluconazole has been the most commonly used.

Objective: To assess the cost effectiveness of itraconazole compared with both fluconazole and no prophylaxis for the prevention of invasive fungal infections in haematological patients, mean age 51 years, in Germany and The Netherlands. Study design: We designed a probabilistic decision model to fully incorporate the uncertainty associated with the risk estimates of acquiring an invasive fungal infection. These risk estimates were extracted from two meta-analyses, evaluating the effectiveness of fluconazole and itraconazole and no prophylaxis. The perspective of the analysis was that of the healthcare sector; only medical costs were taken into account. All costs were reported in $€$, year 2004 values.

Cost effectiveness was expressed as net costs per invasive fungal infection averted. No discounting was performed, as the model followed patients during their neutropenic period, which was assumed to be less than 1 year.

Results: According to our probabilistic decision model, the monetary benefits of averted healthcare exceed the costs of itraconazole prophylaxis under baseline assumptions (95\% CI: from cost-saving to $€ 5000$ per invasive fungal infection averted). Compared with fluconazole, itraconazole is estimated to be both more effective and more economically favourable, with a probability of almost $98 \%$. 
Conclusions: In specific groups of neutropenic patients treated for haematological malignancies, itraconazole prophylaxis could potentially reduce overall healthcare expenditure, without harming effectiveness, in settings where fluconazole is common practice in the prophylaxis of invasive fungal infections.

Fungal infections are frequent complications in neutropenic patients treated for haematological malignancies. There has been a large increase in the incidence of invasive fungal infections over the last 2 decades as a result of the increased number of patients who become immunocompromized as a result of chemotherapy or the underlying disease. ${ }^{[1,2]}$ In Europe and North America, the main causative agents are Aspergillus and Candida species. The incidence rate of these fungal infections depends, among other things, on the duration of neutropenia, the underlying disease and the chemotherapy given. ${ }^{[3,4]}$

As invasive fungal infections are associated with a high mortality rate, early treatment is required. ${ }^{[5]}$ However, the diagnosis of invasive fungal infections is difficult. ${ }^{[6]}$ Therefore, effective antifungal prophylaxis is desirable in high-risk patients to reduce morbidity and mortality. So far, the main antifungal agent used for prophylaxis in Europe has been fluconazole. However, a major shortcoming of fluconazole is that it lacks activity against Aspergillus species and many non-albicans Candida species. ${ }^{[7]}$ An alternative prophylactic antifungal option is itraconazole, which has activity against most Candida species as well as Aspergillus species. ${ }^{[8]}$

In order to aid decisions concerning which intervention provides the best value for limited healthcare resources for antifungal prophylaxis, it is important to assess the relative cost effectiveness of each treatment. As there are no published data comparing the cost effectiveness of these antifungal agents, the primary objective of this study was to assess the cost effectiveness of itraconazole compared with fluconazole for the prevention of inva- sive fungal infections in immunocompromized patients. In addition, itraconazole was compared with an option of 'no prophylaxis'. As probabilistic analysis has became the state-of-the-art in cost-effectiveness analysis, a probabilistic decision-analytic model was used. This cost-effectiveness analysis was carried out for two different countries: Germany and The Netherlands.

\section{Methods}

A probabilistic decision-tree model was designed to evaluate the cost effectiveness of itraconazole for the prophylaxis of Candida and Aspergillus infections. The standard pharmacotherapeutic strategy of fluconazole and another common strategy of 'no prophylaxis' were chosen as comparators; other antimycotic drugs, such as the newer agents voriconazole and caspofungin, are not yet registered for the prophylaxis of systemic fungal infection. The clinical management and diagnosis of invasive fungal infections in neutropenic patients is complicated. As with all models, our model essentially provides a simplified representation of reality, while still grasping the most crucial factors in order to validly evaluate the relative cost effectiveness of the antifungal options.

\section{Decision Model}

We designed a probabilistic model with probability distributions for the important parameters. The probabilistic decision model was constructed in Treeage Data $\mathrm{PRO}^{\mathrm{TM}}$, version 4.0. The decision model followed a population of patients treated for haematological malignancies during their neutropenic period (figure 1). The model provided the 
following three treatment pathways: (i) no prophylaxis; (ii) fluconazole prophylaxis; and (iii) itraconazole prophylaxis. The probability of invasive fungal infections in the three arms was taken from studies included in two meta-analyses evaluating the effectiveness of fluconazole and itraconazole. ${ }^{[7,8]}$ As in the two meta-analyses, we did not make a distinction for the type of Candida and Aspergillus infection.

As there is heterogeneity in the trials with regard to the patient population (e.g. regarding high- and low-risk groups or underlying disease) and study design, we used the random effect method of DerSimonian and Laird ${ }^{[9,10]}$ to combine the results of the different studies. Baseline risks (calculated as the number of patients with a certain event divided by the total number of patients) for invasive fungal infections in the absence of prophylaxis were extracted from seven studies comparing high-dose fluconazole (400 mg orally or $200 \mathrm{mg}$ intravenously [IV]) with placebo or no therapy. ${ }^{[1-18]}$ For the model, only probable or proven infections were taken into account. Distributions used for baseline risks were assumed to be either Normal or triangular (when three times the standard deviation exceeded the mean), as proportions are by definition positive (table I). Relative risks for invasive fungal infections during the use of fluconazole or itraconazole prophylaxis compared with no prophylaxis were assumed to follow a lognormal distribution (table I). ${ }^{[19]}$

For fluconazole, the relative risks for invasive fungal infections compared with no prophylaxis were extracted from the previously mentioned seven studies. ${ }^{[11-18]}$ Accordingly, transition probabilities for the fluconazole arm were obtained by multiplying the baseline risks for invasive fungal infections with these relative risk estimates. Likewise, risks for invasive fungal infections during the use of itraconazole prophylaxis compared with fluconazole prophylaxis were obtained by extracting relative risks from three studies comparing itraconazole in a bioavailable dose of at least $200 \mathrm{mg} /$ day with highdose fluconazole ${ }^{[20-22]}($ table I).

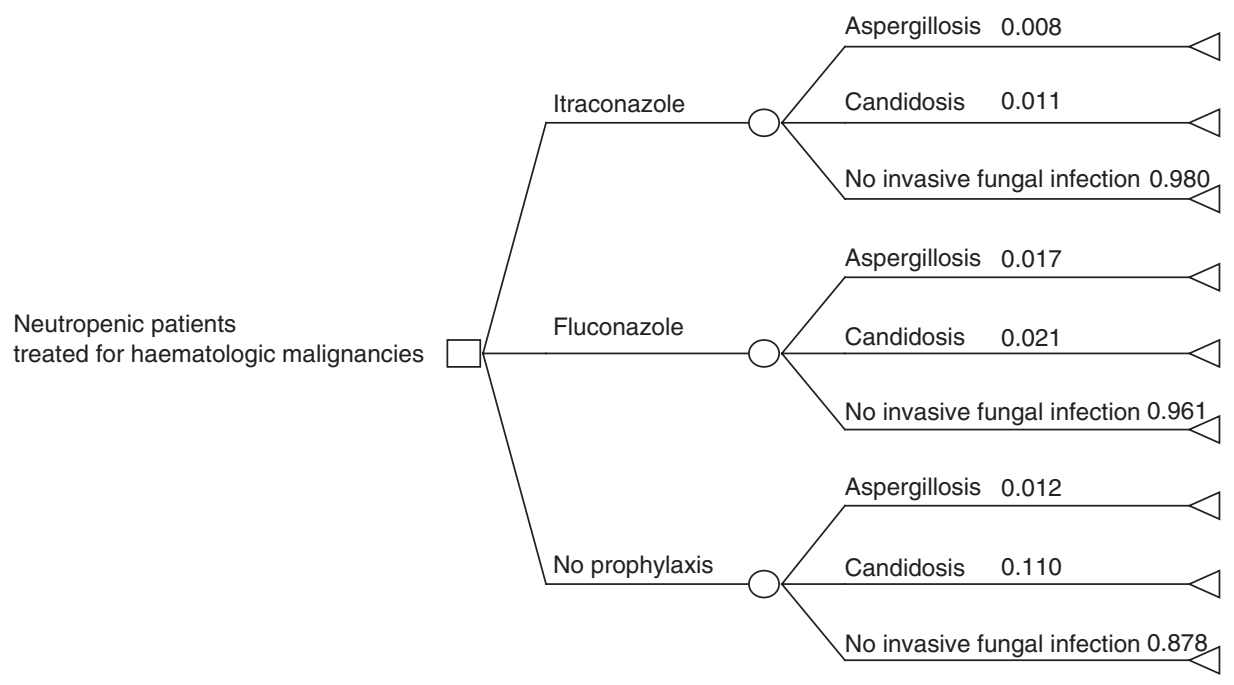

Fig. 1. Decision model with the accompanying (rounded) mean transition probabilities for prophylactic itraconazole, fluconazole or no prophylaxis for invasive fungal infection in neutropenic patients with haematological malignancies. Probabilities of 'no invasive fungal infection' were obtained by subtracting from 'one' both the probability of candidosis [p(candidosis)] and the probability of aspergillosis [p(aspergillosis)] \{i.e. 1-p(candidosis) - $p$ (aspergillosis) $\}$. 
Table I. Distributions used for the transition probabilities in the decision model of prophylactic itraconazole, fluconazole or no prophylaxis for neutropenic patients with haematological malignancies

\begin{tabular}{|c|c|c|c|c|c|c|c|}
\hline \multirow[t]{2}{*}{ Prophylaxis } & \multirow[t]{2}{*}{ Infection } & \multirow[t]{2}{*}{ Distribution $^{a}$} & \multicolumn{4}{|c|}{ Parameters } & \multirow[t]{2}{*}{ References } \\
\hline & & & $\mu$ & SE $(\mu)$ & $\mathrm{RR}$ & $\mathrm{SE}(\operatorname{lnRR})$ & \\
\hline No prophylaxis & Candidosis & Normal & 0.110 & $6.62 \times 10^{-4}$ & & & $11-18$ \\
\hline No prophylaxis & Aspergillosis & Triangular ${ }^{b}$ & 0.012 & $1.74 \times 10^{-5}$ & & & $11-18$ \\
\hline Fluconazole & Candidosis & Lognormal & & & $0.194^{c}$ & 0.070 & $11-18$ \\
\hline Fluconazole & Aspergillosis & Lognormal & & & $1.39^{c}$ & 0.134 & $11-18$ \\
\hline Itraconazole & Candidosis & Lognormal & & & $0.524^{d}$ & 0.207 & $20-22$ \\
\hline Itraconazole & Aspergillosis & Lognormal & & & $0.486^{d}$ & 0.097 & $20-22$ \\
\hline \multicolumn{8}{|c|}{ a For the rationale of the choice of the distributions see text. } \\
\hline \multicolumn{8}{|c|}{ b $\quad$ Minimum $=0$, maximum $=0.018$, mode $=0.017$} \\
\hline \multicolumn{8}{|c|}{ c RR: fluconazole prophylaxis compared with no prophylaxis. } \\
\hline \multicolumn{8}{|c|}{ d RR: itraconazole prophylaxis compared with fluconazole prophylaxis. } \\
\hline \multicolumn{8}{|c|}{$\mu=$ mean; $\mathbf{I n R R}=$ log relative risk; $\mathbf{R} \mathbf{R}=$ relative risk; $\mathbf{S E}=$ standard error. } \\
\hline
\end{tabular}

Finally, the probabilities of 'no invasive fungal infection' were obtained by subtracting from 'one' both the probability of candidosis [p(candidosis)] and the probability of aspergillosis [p(aspergillosis)] \{i.e. $1-p$ (candidosis $)-p($ aspergillosis $)\}$. In summary, ten studies were used to estimate the risk for an invasive fungal infection after antifungal prophylaxis with itraconazole, fluconazole or no prophylaxis.

\section{Costs}

Both the costs of antifungal prophylaxis and the direct hospital costs associated with the diagnosis, therapy and increased length of stay (LOS) due to an invasive fungal infection were considered. All costs were reported in $€$, year 2004 values.

A retrospective cohort study using data for Germany and The Netherlands was carried out to estimate the mean increased LOS as a result of an invasive fungal infection in neutropenic patients with haematological malignancies.

The German dataset consisted of 178 neutropenic patients with haematological malignancies (International Classification of Diseases - 10th edition [ICD-10] codes: C92.0, C91.0, C95.0, C90.0, C81.9 and C83.9). Of these patients, 41 had breakthrough invasive fungal infections (36 Aspergillus, 4 Candi$d a$ and 1 Mucor) after receiving itraconazole prophylaxis, and were matched with 81 reference patients (two for each case, except in one case where only one adequate match could be found) on underlying disease and time of treatment. The infections were either probable or proven. The LOS was calculated from the first day of chemotherapy until discharge from hospital, or until the start of the second course of chemotherapy.

For The Netherlands, 29 cases (24 Aspergillus and 5 Candida) and 58 reference patients were obtained from the Dutch Prismant-database; cases and reference patients were matched on ICD-9 diagnosis (204.0 and 205.0) and gender. ${ }^{[23]}$ The LOS was calculated from the day of admission until discharge.

Cases and references derived from the two datasets were combined to obtain one estimate of the increased LOS associated with an invasive fungal infection. In this analysis, no distinction was made between the causative agents as there were too few Candida and other infections diagnosed.

Estimated resource use associated with diagnosis and treatment practices for invasive fungal infections in neutropenic patients treated for haemato- 
logical malignancies were derived from expert clinical opinion. This was based on an independent international panel consisting of three clinicians, one from the UK, Germany and The Netherlands. These three experts were chosen based on their expertise in the field. The clinicians completed a detailed questionnaire on the diagnosis and typical treatment of a Candida or Aspergillus infection. We considered the individual answers rather than seeking consensus, to enable sensitivity analysis of differences between experts and countries.

As the decision model is a simplification of complex actual practice, only the main standard diagnostic tests were included in the economic analysis. Therefore, based on the clinician survey, it was assumed that a bronchoscopy, two CT scans and 13 x-rays (first week every day and thereafter twice a week) would be performed for each Aspergillus infection, and seven diagnostic blood cultures would be undertaken for each Candida and Aspergillus infection.

Treatment practices for invasive fungal infections as determined by the expert panel in the questionnaire are shown in table II. With respect to the treatment options, we averaged the proposed treatment schemes to get one estimate that was a good representation of the average treatment across the UK, Germany and The Netherlands. We note that the result is a momentary estimate, which by necessity is liable to change in response to new information and the availability of new drugs. The treatment was assumed to be IV for 10 days and oral for the remaining 20 days. However, as amphotericin B liposomal (Ambisome ${ }^{\circledR}$ 1) and caspofungin only exist in the IV-administration form, treatment with these two antifungal agents was assumed to be IV for the full period of 30 days. Potential differences in effectiveness between the treatment options were not taken into account in the model.
Finally, national medical unit costs for antifungal treatment, diagnostic tests and hospital stay were linked to the resources used to estimate the average cost per Candida or Aspergillus infection ${ }^{[24-29]}$ (table III).

\section{Cost-Effectiveness Analysis}

The incremental cost effectiveness of itraconazole prophylaxis compared with both fluconazole prophylaxis and no prophylaxis was estimated. The incremental cost-effectiveness ratio (ICER), which was used as the outcome measure, is defined by equation 1:

$$
\operatorname{ICER}=\frac{\Delta \mathrm{C}}{\Delta \mathrm{E}}=\frac{\mathrm{C}_{\mathrm{I}}-\mathrm{C}_{\mathrm{A}}}{\mathrm{E}_{\mathrm{I}}-\mathrm{E}_{\mathrm{A}}}=\frac{\mathrm{C}_{\mathrm{I}}-\mathrm{C}_{\mathrm{A}}}{\left(1-\mathrm{R}_{\mathrm{I}}\right)-\left(1-\mathrm{R}_{\mathrm{A}}\right)}
$$

(Eq. 1)

where $C_{I}$ and $C_{A}$ are the mean costs per patient in, respectively, the itraconazole group and the alternative strategy (fluconazole or no prophylaxis) group. $\mathrm{R}_{\mathrm{I}}$ and $\mathrm{R}_{\mathrm{A}}$ represent the mean risk for an invasive fungal infection per patient in each group. From this, effectiveness (E) is defined as the estimated probability for the absence of an invasive fungal infection. Accordingly, cost effectiveness was expressed as net costs per invasive fungal infection averted. The perspective for the analysis was that of the healthcare sector, as only direct medical costs were taken into account. Discounting future costs and health outcomes was not applicable, as the model followed patients during their neutropenic period, which was assumed to be $<1$ year.

In the baseline analysis, we performed two analyses to estimate the cost effectiveness of itraconazole prophylaxis for Germany and The Netherlands separately. For each analysis, we conducted 10000 Monte Carlo simulations to fully incorporate the uncertainty associated with the estimated transition probabilities (second order uncertainty). ${ }^{[30]}$ The results of the Monte Carlo simulations were presented

1 The use of trade names is for product identification purposes only and does not imply endorsement. 


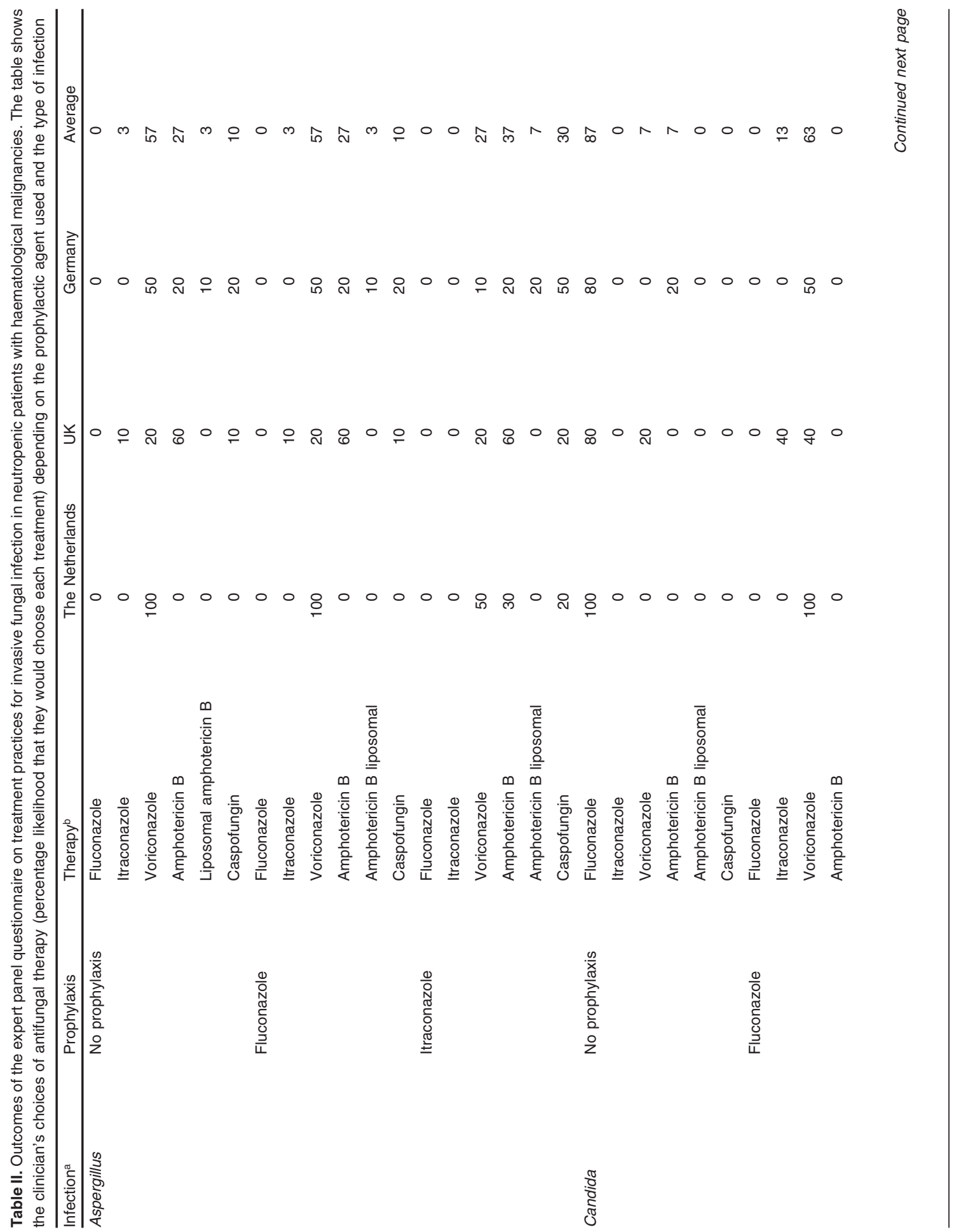




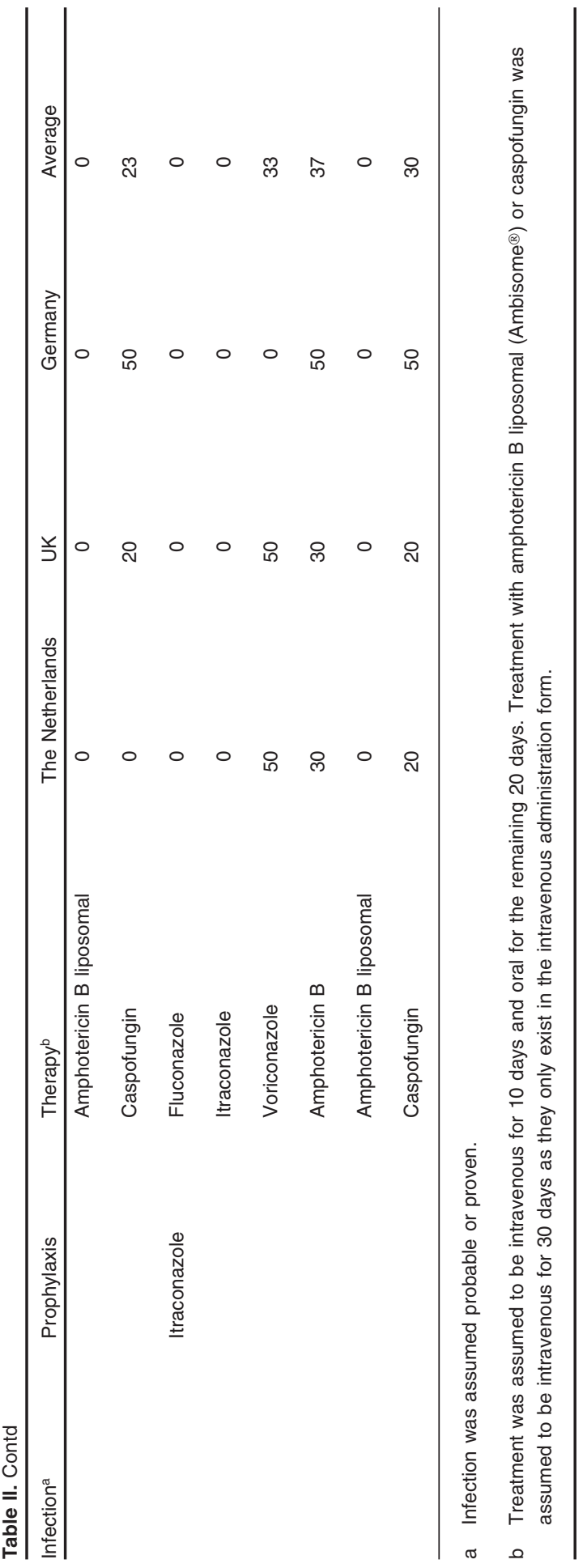

in a cost-effectiveness plane (CE plane) ${ }^{\left[{ }^{[1]}\right.} \mathrm{A}$ dominant therapy is clearly to be preferred (southeast quadrant of the CE plane). If a therapy is more effective and higher cost (northeast quadrant of the CE plane), the adoption decision depends on the maximum willingness to pay of decision makers for each unit of additional benefit. In The Netherlands, decision makers use an informal threshold of $€ 20000$ per life-year gained, while for Germany such an explicit threshold does not currently exist. ${ }^{[32]}$

\section{Sensitivity Analyses}

To further evaluate the level of uncertainty in the outcomes, a univariate sensitivity analysis (first order uncertainty) was performed. ${ }^{[30]}$ As the clinical management of invasive fungal infections is complex and treatment practices differ widely between different countries and different hospitals, the impact on the cost-effectiveness results of different treatment schemes was assessed. For Germany, we performed an analysis in which we only used the treatment scheme as suggested by the German expert and likewise for The Netherlands.

Furthermore, we investigated sensitivity regarding incidences for aspergillosis and candidosis and treatment modalities for the two invasive fungal infections regarding LOS and treatment duration.

The incidences of invasive fungal infections differ widely among different institutions and, in general, the incidence of Aspergillus infections has been increasing in recent years. We took this into account in the sensitivity analysis by using an equal infection rate for aspergillosis and candidosis (i.e. 0.110 in the absence of prophylaxis).

Because of the limited number of candidosis hospitalizations in our data $(n=9)$, we were not able to make a reliable distinction in the LOS between Aspergillus and Candida infections. In the sensitivity analysis, we investigated the potential differences in LOS that might exist between the two infections 
Table III. Costs (€, year 2004 values) for hospital stay, diagnostic tests and antifungal treatment for invasive fungal infection in The Netherlands and Germany

\begin{tabular}{|c|c|c|c|}
\hline Resource & The Netherlands & Germany $^{a}$ & References \\
\hline \multicolumn{4}{|l|}{ Diagnostics } \\
\hline Bronchoscopy & 351.37 & 38.89 & 25,29 \\
\hline Blood culture & 25.19 & 20.95 & 25,29 \\
\hline CT-scan & 188.91 & 142.50 & 25,29 \\
\hline X-ray & 44.92 & 27.88 & 25,29 \\
\hline \multicolumn{4}{|l|}{ Hospital stay } \\
\hline Standard care (day) & 359.24 & 358.91 & 25,27 \\
\hline Intensive care (day) & 1684.00 & 984.96 & 25,26 \\
\hline \multicolumn{4}{|l|}{ Therapy $^{b}$} \\
\hline Fluconazole PO (400 mg) & 24.19 & 21.63 & 24,28 \\
\hline Fluconazole IV (400 mg) & 37.49 & 69.14 & 24,28 \\
\hline Itraconazole PO (400 mg) & 13.52 & 13.44 & 24,28 \\
\hline Itraconazole IV (250 mg) & 189.50 & 188.00 & 24,28 \\
\hline Voriconazole PO (400 mg) & 60.09 & 114.77 & 24,28 \\
\hline Voriconazole IV (400 mg) & 268.00 & 208.07 & 24,28 \\
\hline Amphotericin B IV (50 mg) & 13.56 & 83.42 & 24,28 \\
\hline Amphotericin B liposomal IV (400 mg) & 780.80 & 770.43 & 24,28 \\
\hline Caspofungin IV (50 mg) & 478.00 & 605.10 & 24,28 \\
\hline \multicolumn{4}{|l|}{ Total mean cost } \\
\hline Aspergillus infection ${ }^{c}$ & $11993-14613$ & $12036-17070$ & \\
\hline Candida infection ${ }^{c}$ & $7207-11893$ & $6008-11742$ & \\
\hline \multicolumn{4}{|c|}{ a For Germany we assumed that $90 \%$ were socially insured and $10 \%$ were privately insured. } \\
\hline \multicolumn{4}{|c|}{ b Costs per day based on the doses per day given in the brackets. } \\
\hline \multicolumn{4}{|c|}{ c Depending on the exact types of prophylaxis and subsequent treatment (see table II). } \\
\hline \multicolumn{4}{|l|}{ IV = intravenous; $\mathbf{P O}=$ oral. } \\
\hline
\end{tabular}

by using unequal additional LOS for Candida and Aspergillus infections as suggested in the literature. Wilson et al. ${ }^{[33]}$ estimated a ratio for additional LOS for a Candida infection versus an Aspergillus infection at $14: 19$. In our retrospective study, the excess LOS was estimated at 11.11 days when only patients with Aspergillus infection were considered. In the sensitivity analysis, this value was input as the excess LOS due to Aspergillus infection. We then applied the ratio of Wilson et al. ${ }^{[33]}$ to obtain an unequal estimate of 8.19 days for the excess LOS as a result of a Candida infection. We assumed 1 day and 2 days of intensive care for a Candida and an Aspergillus infection, respectively.

We also investigated the effect of unequal treatment lengths for the two types of invasive fungal infections. Torfs ${ }^{[34]}$ noted that the mean duration of antifungal treatment for aspergillosis is considerably longer than that of candidosis. In the sensitivity analysis, we thus prolonged the treatment duration from 30 days up to 40 days in the case of an Aspergillus infection. 


\section{Results}

Increased Length of Stay for Invasive Fungal Infection

The mean age was 50 years (range 14-71 years, $\mathrm{n}=139$ ) for the control patients and 53 years (range 14-71 years, $n=70$ ) for patients with an invasive fungal infection. Forty percent of the patients in the reference group and $37 \%$ in the case group were female. Most of the patients had acute leukaemia $(>95 \%)$. The mean LOS for patients with an invasive fungal infection was $45 \pm$ SD20.8 days, whereas the mean LOS for control patients was $36 \pm 12.5$ days. Therefore, the mean increase in LOS associated with an invasive fungal infection in a neutropenic patient treated for haematological malignancy is estimated as 9.3 days $(95 \%$ CI $4.5,13.6)$. It was assumed that the increased LOS consisted of 2 days in an ICU and the remainder in standard care. ${ }^{[35]}$

\section{Baseline Analysis}

The results of the 10000 Monte Carlo simulations covering the baseline analysis are given in table IV. The mean cost per patient for prophylaxis and the direct medical costs associated with an invasive fungal infection was lower for the itraconazole arm (The Netherlands: $€ 780$, Germany: $€ 785$ ) than for both the fluconazole arm (The Netherlands: $€ 1172$, Germany: €1146) and the no prophylaxis arm (The Netherlands: €928, Germany: €791). The mean risk of an invasive fungal infection was lowest in patients receiving itraconazole prophylaxis $(2.2 \%)$, while that risk was almost doubled in the fluconazole group (4.0\%). Patients who did not receive any prophylaxis had a risk of $12.1 \%$ for an invasive fungal infection. The risk estimates extracted from the studies included in the two metaanalyses, and pooled LOS estimates were applied to both the Dutch and German analyses. Hence, differ- ences in the economic results were due to different national unit costs applied in each country.

As is demonstrated in table $\mathrm{V}$, itraconazole prophylaxis is dominant (i.e. more effective $[\Delta \mathrm{E}>0]$ and cost saving $[\Delta \mathrm{C}<0])$ compared with both fluconazole and a 'no prophylaxis' scenario. The estimated sampling distributions of the ICERs are shown in figure 2 . When comparing itraconazole to no prophylaxis, the $95 \%$ confidence interval ranges from cost saving to approximately $€ 4000$ per invasive fungal infection averted for the Dutch situation, and $€ 5500$ for Germany. Due to difficulties in interpretation of a negative ICER, for the comparison of itraconazole with fluconazole, we determined the proportion of the 10000 Monte Carlo simulations lying within the southeast quadrant of the $\mathrm{CE}$ plane. As almost 0.98 of the outcomes refer to the southeast quadrant, the probability that fluconazole is dominated by itraconazole is almost $98 \%$. For illustrative purposes, such probabilities for the other comparison are also shown in table $\mathrm{V}$.

Additionally, figure 3 presents some detailed information on the costs averted through itraconazole prophylaxis compared with no prophylaxis and fluconazole for The Netherlands. In the former case, averted costs on LOS constitute the major share of savings; in the latter case, averted treatment costs for invasive fungal infections appear to be almost equally important. This illustrates that, in the absence of prophylaxis, relatively cheap antifungals are used for treatment, whereas this not the case if itraconazole or fluconazole prophylaxis is chosen. Similar pie charts were found for Germany (not shown here).

\section{Sensitivity Analysis}

Instead of averaging the treatment schemes across countries, applying only the treatment schemes suggested by the Dutch expert for the Dutch analysis and the German expert for the German analysis had only a limited effect on the out- 
Table IV. Costs (€, year 2004 values) and risk of invasive fungal infection with prophylactic itraconazole, fluconazole or no prophylaxis for neutropenic patients with haematological malignancies. Results based on 10000 Monte Carlo simulations

\begin{tabular}{|c|c|c|c|}
\hline Parameter & Itraconazole & Fluconazole & No prophylaxis \\
\hline \multicolumn{4}{|l|}{ Baseline analysis } \\
\hline \multicolumn{4}{|l|}{ The Netherlands } \\
\hline mean cost per patient & 780 & 1172 & 928 \\
\hline mean risk of invasive fungal infection & 0.022 & 0.040 & 0.121 \\
\hline \multicolumn{4}{|l|}{ Germany } \\
\hline mean cost per patient & 785 & 1146 & 791 \\
\hline mean risk of invasive fungal infection & 0.022 & 0.040 & 0.121 \\
\hline \multicolumn{4}{|l|}{ Sensitivity analysis } \\
\hline \multicolumn{4}{|l|}{ Country-specific treatment schemes } \\
\hline \multicolumn{4}{|l|}{ The Netherlands } \\
\hline mean cost per patient & 751 & 1112 & 904 \\
\hline mean risk of invasive fungal infection & 0.022 & 0.040 & 0.122 \\
\hline \multicolumn{4}{|l|}{ Germany } \\
\hline mean cost per patient & 863 & 1280 & 815 \\
\hline mean risk of invasive fungal infection & 0.022 & 0.039 & 0.121 \\
\hline \multicolumn{4}{|c|}{$\begin{array}{l}\text { Differential excess LOS according to type of invasive fungal } \\
\text { infection }^{\text {a }}\end{array}$} \\
\hline \multicolumn{4}{|l|}{ The Netherlands } \\
\hline mean cost per patient & 765 & 1146 & 750 \\
\hline mean risk of invasive fungal infection & 0.022 & 0.040 & 0.121 \\
\hline \multicolumn{4}{|l|}{ Germany } \\
\hline mean cost per patient & 777 & 1133 & 693 \\
\hline mean risk of invasive fungal infection & 0.022 & 0.039 & 0.121 \\
\hline \multicolumn{4}{|c|}{ Equal incidence of the two types of invasive fungal infection ${ }^{\mathrm{b}}$} \\
\hline \multicolumn{4}{|l|}{ The Netherlands } \\
\hline mean cost per patient & 1862 & 2914 & 2104 \\
\hline mean risk of invasive fungal infection & 0.096 & 0.185 & 0.219 \\
\hline \multicolumn{4}{|l|}{ Germany } \\
\hline mean cost per patient & 1859 & 2744 & 1867 \\
\hline mean risk of invasive fungal infection & 0.096 & 0.185 & 0.220 \\
\hline \multicolumn{4}{|l|}{ Longer treatment duration for aspergillosis ${ }^{c}$} \\
\hline \multicolumn{4}{|l|}{ The Netherlands } \\
\hline mean cost per patient & 800 & 1190 & 947 \\
\hline mean risk of invasive fungal infection & 0.022 & 0.040 & 0.122 \\
\hline \multicolumn{4}{|l|}{ Germany } \\
\hline mean cost per patient & 809 & 1171 & 807 \\
\hline mean risk of invasive fungal infection & 0.022 & 0.039 & 0.121 \\
\hline \multicolumn{4}{|c|}{$\begin{array}{l}11.11 \text { days for an Aspergillus infection and } 8.19 \text { days for a Candida infection. We assumed } 1 \text { day and } 2 \text { days of intensive care for a } \\
\text { Candida and an Aspergillus infection, respectively. }\end{array}$} \\
\hline \multicolumn{4}{|c|}{ b An infection rate of 0.110 in the absence of prophylaxis. } \\
\hline \multicolumn{4}{|c|}{$\begin{array}{l}\text { c The treatment duration was extended from } 30 \text { days to } 40 \text { days in the case of an Aspergillus infection. } \\
\text { LOS = length of stay. }\end{array}$} \\
\hline \multicolumn{4}{|l|}{ LOS = length of stay. } \\
\hline
\end{tabular}


Table V. Estimated incremental cost-effectiveness ratios (ICERs) for prophylactic itraconazole versus fluconazole and versus no prophylaxis for invasive fungal infections in neutropenic patients with haematological malignancies. The ICER is the net costs (€, year 2004 values) per invasive fungal infection averted

\begin{tabular}{|c|c|c|c|c|c|}
\hline \multirow[t]{2}{*}{ Parameter } & \multicolumn{2}{|c|}{ Itraconazole vs fluconazole } & \multicolumn{3}{|c|}{ Itraconazole vs no prophylaxis } \\
\hline & ICER & $\begin{array}{l}\% \text { of simulations } \\
\text { that produced a } \\
\text { CS result }{ }^{\mathrm{a}}\end{array}$ & ICER & $\begin{array}{l}\text { Upper } \mathrm{Cl} \text { limit of } \\
\text { ICER }^{\mathrm{b}}\end{array}$ & $\begin{array}{l}\% \text { of simulations } \\
\text { that produced a } \\
\text { CS result }\end{array}$ \\
\hline \multicolumn{6}{|l|}{ Baseline analysis } \\
\hline The Netherlands & $\mathrm{CS}^{\mathrm{c}}$ & 97.63 & CS & 3972 & 79.47 \\
\hline Germany & CS & 97.66 & CS & 5474 & 52.33 \\
\hline \multicolumn{6}{|c|}{ Sensitivity analysis } \\
\hline \multicolumn{6}{|c|}{ Country-specific treatment schemes } \\
\hline The Netherlands & CS & 97.57 & CS & 3943 & 80.64 \\
\hline Germany & CS & 97.51 & 485 & 7198 & 40.62 \\
\hline \multicolumn{6}{|c|}{$\begin{array}{l}\text { Differential excess LOS according to type of } \\
\text { invasive fungal infection }\end{array}$} \\
\hline The Netherlands & CS & 97.63 & 150 & 5881 & 46.68 \\
\hline Germany & CS & 97.74 & 842 & 6675 & 28.76 \\
\hline \multicolumn{6}{|c|}{$\begin{array}{l}\text { Equal incidence of the two types of invasive } \\
\text { fungal infection }\end{array}$} \\
\hline The Netherlands & CS & 98.39 & CS & 154068 & 72.35 \\
\hline Germany & CS & 98.43 & CS & 191301 & 59.26 \\
\hline \multicolumn{6}{|c|}{ Longer treatment duration for aspergillosis } \\
\hline The Netherlands & CS & 97.64 & CS & 4178 & 78.04 \\
\hline Germany & CS & 97.97 & 26 & 6142 & 50.13 \\
\hline \multicolumn{6}{|c|}{ a Upper $95 \%$ Cls of the ICER are also CS. Thus, only the percentage of Monte Carlo simulations that refer to CSs is presented. } \\
\hline \multicolumn{6}{|c|}{ b Only the upper $95 \%$ Cls are given because the lower limits always indicated dominance (i.e. CS and more effective); see figure 2 . } \\
\hline \multicolumn{6}{|c|}{$\begin{array}{l}\text { c Where itraconazole is CS (i.e. both more effective and less costly than the alternative) ICER values are negative and therefore not } \\
\text { useful. }\end{array}$} \\
\hline
\end{tabular}

comes (tables IV and V). For The Netherlands, itraconazole remained dominant to no prophylaxis, whilst for Germany there was an incremental cost of $€ 485$ to avert an invasive fungal infection compared with no prophylaxis. For both countries, itraconazole remained dominant to fluconazole.

The likely difference in LOS between Aspergillus and Candida infections was investigated in the sensitivity analysis. An unequal additional LOS of 11.11 days for aspergillosis and 8.19 days for candidosis, along with an assumed reduced time in intensive care with candidosis ( 1 day vs 2 days with aspergillosis), was input into the model. This was associated with a small decrease in the mean cost per patient in all three prophylaxis arms (table IV). This also resulted in slightly positive incremental costs per infection averted for itraconazole versus no prophylaxis (The Netherlands: $€ 150$ per infection averted, Germany: $€ 842$ per infection averted; table $\mathrm{V})$. Itraconazole remained dominant to fluconazole in both countries.

Furthermore, we investigated the effect of unequal treatment lengths for the two types of infections by prolonging the treatment duration from 30 days up to 40 days in the case of an Aspergillus infection. This had only a small effect on the out- 
a

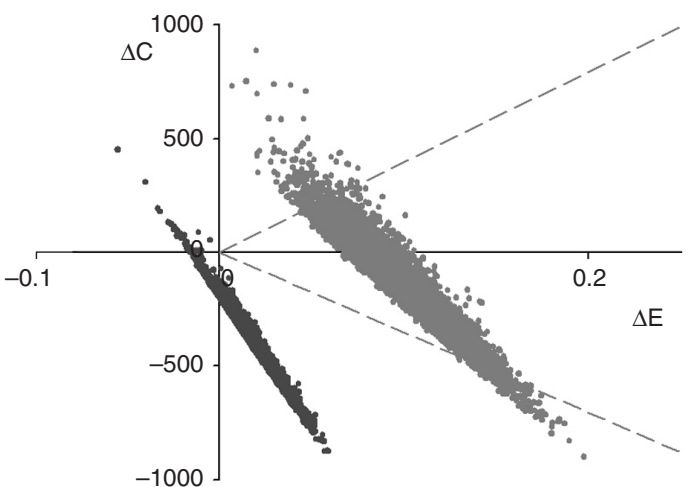

b

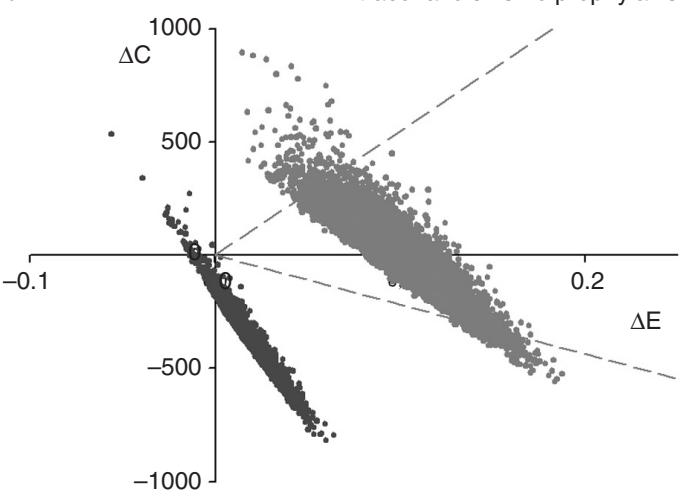

Fig. 2. Results of the baseline analysis of prophylactic itraconazole, fluconazole or no prophylaxis for invasive fungal infection in neutropenic patients with haematological malignancies for (a) The Netherlands and (b) Germany. Results are based on 10000 Monte Carlo simulations. Dashed lines represent the limits of the $95 \%$ confidence intervals of itraconazole compared with no prophylaxis. $\Delta \mathbf{C}=$ change in costs ( $€$, year 2004 values); $\Delta \mathbf{E}=$ change in effectiveness (number of invasive fungal infections averted).

comes (table IV); the mean cost per infection averted was slightly increased. In the German situation, this led to an incremental cost per invasive fungal infection averted of $€ 26$ for itraconazole versus no prophylaxis.

Finally, because the incidences of invasive fungal infections differ widely among different institutions, we investigated the effect of assuming equal infection rates for aspergillosis and candidosis (i.e. 0.110 in the absence of prophylaxis) in the sensitivity analysis. This led to a considerable increase in a

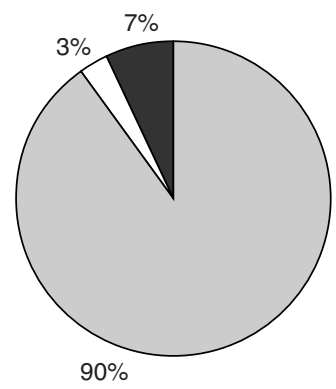

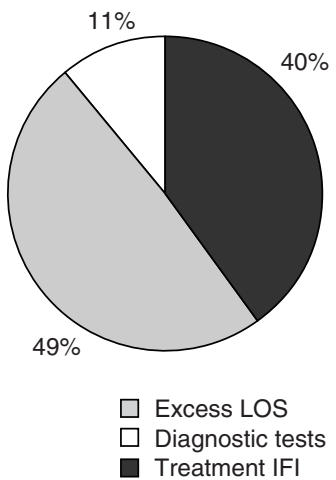

Fig. 3. Percentage distribution of costs averted by cost category for (a) itraconazole versus no prophylaxis and (b) itraconazole versus fluconazole. IFI = invasive fungal infection; LOS = length of stay. both the mean costs and the mean risk for an invasive fungal infection (tables IV and V). Itraconazole became more dominant compared with fluconazole in both countries. Compared with no prophylaxis, itraconazole became more favourable on average as well. However, the upper 95\% confidence limits were increased $(>€ 150$ 000) due to an increased variation in the results.

\section{Discussion}

We present the first study comparing the cost effectiveness of itraconazole and fluconazole in the prophylaxis of invasive fungal infections in neutropenic patients treated for haematological malignancies. According to our probabilistic decision model, the monetary benefits of averted healthcare exceed the costs of itraconazole prophylaxis under the baseline assumptions; itraconazole is estimated to be cost saving compared with no prophylaxis for The Netherlands (95\% CI: from cost saving to a cost of $€ 3972$ per invasive fungal infection averted) and Germany (95\% CI: from cost saving to $€ 5474$ per invasive fungal infection averted). For both countries, itraconazole is also estimated to be both more effective and more economically favourable than 
fluconazole. The probability that itraconazole dominates fluconazole was estimated at almost $98 \%$.

For estimating the hospital costs associated with an invasive fungal infection, we first performed a retrospective cohort study to determine the increased LOS. The increased LOS was estimated at 9.3 days (95\% CI 4.5, 13.6). This estimate is slightly lower than the 11.3 days estimated by Menzin et al. ${ }^{[35]}$ They estimated the increased LOS associated with serious fungal infections among elderly patients diagnosed with acute myeloid leukaemia. Important differences, which could, among other things, explain the difference in outcome between the studies were the mean age of the cohorts and the baseline LOS of the reference group. In the study conducted by Menzin et al., ${ }^{[35]}$ the average age of patients in the study cohort was 73 years, and the baseline LOS of the reference group was 19 days, as compared with 51 years and 36 days in our analysis, respectively.

Sensitivity analyses revealed that varying the key parameters associated with cost estimation (i.e. treatment practices obtained from the questionnaires, treatment duration and excess LOS) had limited impact on the cost effectiveness of itraconazole. As the clinical management of invasive fungal infections differs widely among different hospitals, even within the same country, we performed a sensitivity analysis on the treatment practices of invasive fungal infections. We used the treatment practice as suggested by the Dutch expert for the Dutch analysis and likewise for Germany. With the exception of the German analysis of itraconazole compared with fluconazole (with an incremental cost of $€ 485$ per invasive fungal infection averted), itraconazole was still estimated to be cost saving compared with both fluconazole and no prophylaxis. Furthermore, a longer excess LOS was assumed for an Aspergillus infection. Here, the costs of itraconazole prophylaxis exceeded the monetary benefits of averted healthcare, producing an incremental cost of $€ 150$ and
$€ 842$ per case of invasive fungal infection averted for The Netherlands and Germany, respectively. This means, for The Netherlands, that the prevention of an invasive fungal infection should at least result in a gain of 3 life-days for the ICER to stay below the informal threshold used for assessing value for money for national reimbursement purposes of $€ 20000$ per life-year gained. ${ }^{[32]}$ This seems a very moderate gain as invasive fungal infections are associated with a high mortality rate. ${ }^{[5]}$

Moreover, extending the duration of antifungal treatment for an Aspergillus infection appeared to have only minor influence on the outcomes. However, the infection rate did have a considerable effect on the costs and the effects. This could easily be explained by the fact that the cost effectiveness of preventive strategies such as antifungal prophylaxis always largely depends on the incidence of the particular disease being treated for. Here, the increased incidence of aspergillosis on average resulted in more favourable outcomes for itraconazole. Nevertheless, as the variation increased, the upper $95 \%$ confidence limit exceeded the Dutch threshold of $€ 20000$ per life-year gained when itraconazole was compared with no prophylaxis.

A probabilistic decision model was produced to fully incorporate the uncertainty associated with the transition probabilities. Even though it is not always recognized, the handling of uncertainty in cost-effectiveness models is very important for obtaining reliable results. ${ }^{[30]}$ Another important strength of this study is its use of data obtained from not one, but several clinical trials to estimate the transition probabilities in the model. The considerable number of patients in each treatment group led to more reliable and precise estimates of the probabilities of invasive fungal infections, which increases the validity of the results. Nevertheless, it should be mentioned that absolute baseline risks of Candida and Aspergillus infections depend on the setting and can also differ between hospitals within the same coun- 
try. However, as there are no country-specific data for The Netherlands and Germany, we used a weighted mean estimate across the clinical trials used.

\section{Study Limitations}

There are several limitations of our study. Pharmacoeconomic evaluations most often use lifeyears gained and QALYs as the principal outcome measures. However, without survival data for the patients in this study, it was not possible to provide reliable estimates of life-years gained. Moreover, it may be very difficult to determine invasive fungal infections as the cause of death. ${ }^{[36]}$ Therefore, we used averted invasive fungal infections as the outcome measure. Furthermore, several parameters could not be included in this model.

First, possible adverse effects and potential discontinuation of the prophylactic medications were not taken into account. Discontinuation was indirectly taken into account as we used the efficacy from the intention-to-treat analyses in our metaanalysis. We did assume that switching did not lead to an increase in the length of prophylactic treatment and thus the costs. In general, itraconazole and fluconazole are well tolerated. ${ }^{[8]}$ Nevertheless, the discontinuation rate appears to be higher for itraconazole compared with fluconazole, discontinuation being most often due to gastrointestinal problems. ${ }^{[20-22]}$ So, inclusion of discontinuation in the model is likely to have little influence on the cost estimates. The same may hold true for the inclusion of treatment for adverse effects, as the related costs are minor. ${ }^{[37]}$

Second, switching in the treatment of probable or proven invasive fungal infections was not modelled explicitly, as it appeared not to be an issue from the expert interviews. Nevertheless, in daily practice, it obviously may occur. ${ }^{[38]}$ Moreover, the costs of treating possible adverse effects of antifungal treatment was not included.
Third, only invasive fungal infections were modelled. Fluconazole and itraconazole also showed a significant prophylactic effect against superficial fungal infections. ${ }^{[7,20,22]}$ Although the treatment of superficial infections is relatively straightforward and inexpensive, reduction in incidence improves quality of life. By not including superficial infections, the results can be viewed as conservative in favour of no prophylaxis. However, as no estimates of the costs of superficial infections were identified, these costs were not able to be taken into account.

Finally, we note that it is not necessarily true that the morbidity and costs incurred due to an invasive fungal infection after either no prophylaxis or with prophylaxis would be similar, as assumed in our model. Unfortunately, we were not able to make this distinction with regard to the estimates of the excess LOS and treatment duration because the data on these estimates were not stratified according to the prophylactic agent.

\section{Conclusions}

On the basis of data from several clinical trials, an international panel of experts and Dutch and German resource costing, the model suggests that itraconazole is likely to result in improved outcomes and lower costs compared with fluconazole and no prophylaxis for both The Netherlands and Germany. Therefore, in specific groups of neutropenic patients treated for haematological malignancies, itraconazole prophylaxis could potentially reduce overall healthcare expenditure in settings where fluconazole is common practice in the prophylaxis of invasive fungal infections.

\section{Acknowledgements}

This study was supported by a grant from Ortho Biotech. The co-authors from Ortho Biotech had an important contribution in conceiving and planning the project and in specific tasks (e.g. disseminating the questionnaire). The sponsor had no role in the data analysis and interpretation of the results. The authors from academic institutions retained the right to 
publish the data regardless of the outcomes. The sponsor did comment on various versions of the manuscripts, including the final version. Axel Glasmacher and Archie Prentice have previously received grants, honoraria and/or consultancy fees from Ortho Biotech and Pfizer.

\section{References}

1. McNeil MM, Nash SL, Hajjeh RA, et al. Trends in mortality due to invasive mycotic diseases in the United States, 1980-1997. Clin Infect Dis 2001; 33: 641-7

2. Garber G. An overview of fungal infections. Drugs 2001; 61 Suppl. 1: 1-12

3. Prentice HG, Kibbler CC, Prentice AG. Towards a targeted, risk-based, antifungal strategy in neutropenic patients. Br J Haematol 2000; 100: 273-84

4. Uzun O, Anaisse EJ. Antifungal prophylaxis in patients with hematologic malignancies: a reappraisal. Blood 1995; 86: 2063-72

5. Lin SJ, Schranz J, Teutsch SM. Aspergillosis case-fatality rate: systematic review of the literature. Clin Infect Dis 2001; 32: 358-66

6. Rinaldi MG. Problems in the diagnosis of invasive fungal diseases. Rev Infect Dis 1991; 13: 493-5

7. Kanda Y, Yamamoto R, Chizuka A, et al. Prophylactic action of oral fluconazole against fungal infection of neutropenic patients: a meta-analysis of 16 randomized, controlled trials. Cancer 2000; 89: 1611-25

8. Glasmacher A, Prentice A, Gorschlüter M, et al. Itraconazole prevents invasive fungal infections in neutropenic patients treated for hematologic malignancies: evidence from a metaanalysis of 3,597 patients. J Clin Oncol 2003; 21 (24): 4615-26

9. Sutton AJ, Abrams KR, Jones DR, et al. Methods for metaanalysis in medical research. Chichester: John Wiley \& Sons Ltd, 2000

10. DerSimonian R, Laird N. Meta-analysis in clinical trials. Control Clin Trials 1986; 7: 177-88

11. Goodman JL, Winston DJ, Greenfield RA, et al. A controlled trial of fluconazole to prevent fungal infections in patients undergoing bone marrow transplantation. N Engl J Med 1992; 326: $845-51$

12. Winston DJ, Chandrasekar PH, Lazarus HM, et al. Fluconazole prophylaxis of fungal infections in patients with acute leukemia: results of a randomized placebo-controlled, double blind, multi-center trial. Ann Intern Med 1993; 118: 495-503

13. Chandrasekar PH, Gatny CM. Effect of fluconazole prophylaxis on fever and use of amphotericin in neutropenic cancer patients: the Bone Marrow Transplantation Team. Chemotherapy 1994; 40: 136-43

14. Chandrasekar PH, Gathy CM. The effect of fluconazole prophylaxis on fungal colonization in neutropenic cancer patients: the Bone Marrow Transplantation Team. J Antimicrob Chemother 1994; 33: 309-18

15. Schaffner A, Schaffner M. Effect of prophylactic fluconazole on the frequency of fungal infections, amphotericin B use, and health care costs in patients undergoing intensive chemother- apy for hematologic neoplasias. J Infect Dis 1995; 172: 103541

16. Slavin MA, Osborne B, Adams R, et al. Efficacy and safety of fluconazole prophylaxis for fungal infections after bone marrow transplantation: a prospective, randomised, double-blind study. J Infect Dis 1995; 171: 1545-52

17. Kern W, Behre G, Rudolf T, et al. Failure of fluconazole prophylaxis to reduce mortality or the requirement of systemic amphotericin B therapy during treatment for refractory acute myeloid leukemia. Cancer 1998; 83: 289-301

18. Rotstein C, Bow EJ, Laverdiere M, et al. Randomized placebocontrolled trial of fluconazole prophylaxis for neutropenic cancer patients: benefit based on purpose and intensity of cytotoxic therapy. Clin Infect Dis 1999; 28: 331-40

19. Altman DG. Practical statistics for medical research. Boca Raton (FL): CRC Press, 1999

20. Winston DJ, Maziarz RT, Chandrasekar PH, et al. Intravenous and oral itraconazole versus intravenous and oral fluconazole for long-term antifungal prophylaxis in allogeneic hematopoietic stem-cell transplant recipients: a multicenter, randomized trial. Ann Intern Med 2003; 138: 705-13

21. Marr KA, Crippa F, Leisenring W. Itraconazole versus fluconazole for prevention of fungal infections in patients receiving allogeneic stem cell transplants. Transplantation 2004; 103 (4): 1527-33

22. Glasmacher A, Cornely O, Ullman AJ. An open-label randomized trial comparing itraconazole oral solution with fluconazole oral solution for primary prophylaxis of fungal infections in patents with haematological malignancy and profound neutropenia. J Antimicrob Chemother 2006; 57: 317-25

23. Prismant: National LMR information [online and further data specifically requested]. Available from URL: http://cognos server.prismant.nl/cognos7/cgi-bin/ppdscgi.cgi?DC=Q\&E=/ Prisma-Landelijke-LMR/Landelijke+LMR-informatie+-+ Diagnosen [Accessed 2006 Jul 01]

24. Health Care Insurance Board. Dutch pharmacoeconomic guidelines 2004 [in Dutch]. Amstelveen: Health Care Insurance Board, 2003 [online]. Available from URL: http:// www.fk.cvz.nl [Accessed $2006 \mathrm{Jul}$ 01]

25. Oosterink JB, Bouwmans CAM, Koopmanschap MA, et al. Guideline for costing research, methods and standardized prices for economic evaluations in health care. Diemen: Health Care Insurance Board, 2004

26. Neilson AR, Moerer O, Burchardi H, et al. A new concept for DRG-based reimbursement of services in German intensive care units: results of a pilot study. Intensive Care Med 2004; 30 (6): $1220-3$

27. Landesamt für Datenverarbeitung und Statistik NordrheinWestfalen [online]. Available from URL: http://www.lds. nrw.de/presse/pressemitteilungen/2005/pres_020_05.html [Accessed 2006 Jul 01]

28. Rote Liste ${ }^{\circledR}$ : arzneimittelinformationen für Deutschland [online]. Available from URL: http://www.rote-liste.de [Accessed 2006 Jul 01]

29. Arztkosten [online]. Available from URL: http://www.e-bis.de/ goae/defaultFrame.htm [Accessed 2006 Jul 01]

30. Briggs AH. Handling uncertainty in cost-effectiveness models. Pharmacoeconomics 2000; 17 (5): 479-500 
31. Black WC. The CE plane: a graphic representation of costeffectiveness. Med Decis Making 1990; 10 (3): 212-4

32. Bos JM, Postma MJ. Using pharmacoeconomics for policy making: is rational decision making enhanced by applying thresholds for cost-effectiveness? Expert Rev Pharmacoeconomics Outcomes Res 2004; 4 (3): 247-50

33. Wilson LS, Reyes CM, Stolpman M, et al. The direct cost and incidence of systematic fungal infections. Value Health 2002; 5 (1): $26-34$

34. Torfs K. Economic aspects of treatment for fungal infections in cancer patients. Eur J Microbiol Infect Dis 1997; 16: 98-107

35. Menzin J, Lang KM, Friedman M, et al. Excess mortality, length of stay, and costs associated with serious fungal infections among elderly cancer patients: findings from linked SEERMedicare data. Value Health 2005; 8 (2): 140-8

36. Gotzsche PC, Johansen HK. Meta-analysis of prophylactic or empirical antifungal treatment versus placebo or no treatment in patients with cancer complicated by neutropenia. BMJ 1997; 314: 1238-44

37. Rentz AM, Halpern MT, Bowden R. The impact of candidemia on length of hospital stay, outcome, and overall cost of illness. Clin Infect Dis 1998; 27: 781-88

38. Jansen JP, Meis JF, Blijlevens NM, et al. Economic evaluation of voriconazole in the treatment of invasive fungal infections in the Netherlands. Curr Med Res Opin 2005; 12 (10): 1535-46

Correspondence: Dr Robin de Vries, Groningen Research Institute of Pharmacy (GRIP), University of Groningen, Antonius Deusinglaan 1, Groningen, 9713 AV, The Netherlands.

E-mail: robin.de.vries@rug.nl 